\title{
Can stochastic quantization evade the sign problem? - the relativistic Bose gas at finite chemical potential
}

\author{
Gert Aarts \\ Department of Physics, Swansea University, Swansea SA2 8PP, United Kingdom
}

(Dated: October 12, 2008)

\begin{abstract}
A nonperturbative study of field theories with a complex action, such as QCD at finite baryon density, is difficult due to the sign problem. We show that the relativistic Bose gas at finite chemical potential has a sign and 'Silver Blaze' problem, similar to QCD. We then apply stochastic quantization and complex Langevin dynamics to study this theory with nonperturbative lattice simulations. Independence of chemical potential at small and a transition to a condensed phase at large chemical potential are found. Lattices of size $N^{4}$, with $N=4,6,8,10$, are used. We show that the sign problem is severe, however, we find that it has no negative effect using this approach. This improves the prospects of applying stochastic quantization to QCD at nonzero density.
\end{abstract}

PACS numbers: $11.15 . \mathrm{Ha}$ 12.38.Mh

Introduction - Field theories with a complex action are difficult to treat nonperturbatively. Because the weight in the path integral $e^{-S}=\left|e^{-S}\right| e^{i \varphi}$ is not real, standard numerical approaches based on a probability interpretation and importance sampling cannot be applied. This has hindered progress in condensed matter and manybody theories, such as frustrated quantum spin systems and strongly correlated electronic systems away from half filling. An outstanding example is QCD at nonzero baryon density. Several methods have been devised to circumvent the sign problem in QCD: reweighting [1, 2], Taylor series expansion [3, 4], imaginary chemical potential and analytic continuation [5, 6], and the use of the canonical ensemble [7, 8$]$ and the density of states [9]. In general these methods can only be applied in a limited region of the phase diagram (such as high temperature and small chemical potential), suffer from overlap problems, and/or are restricted to small volumes.

At vanishing temperature, so far all methods have failed. It is well-known what is supposed to happen: if $\mu$ is the quark chemical potential (so that the fermion determinant satisfies $\left.\operatorname{det} M(\mu)=[\operatorname{det} M(-\mu)]^{*}\right)$, one expects a transition to a condensed phase (nuclear matter) at a critical chemical potential $\mu_{c} \approx m_{N} / 3$, where $m_{N}$ is the nucleon mass. Below $\mu_{c}$, physical observables should be completely independent of $\mu$ (at strictly zero temperature), even though microscopically $\mu$ explicitly enters in the Boltzmann weight. This exact cancelation is known as the 'Silver Blaze' problem [10] and is intimately tied to the sign problem, as has been demonstrated in Random Matrix Theory [11, 12, 13]. It is an outstanding challenge to probe the QCD phase diagram in the low-temperature region at nonzero chemical potential.

The sign problem in QCD arises not because of the anticommuting nature of fermions. Instead, it is due to the behaviour of the action under complex conjugation when the chemical potential is nonzero. Therefore, this sign problem can also be studied in bosonic field theories with a chemical potential coupled to a conserved charge and an action satisfying $S(\mu)=[S(-\mu)]^{*}$. These theories suffer from exactly the same sign and Silver Blaze problems as QCD. In this Letter we consider the relativistic Bose gas at finite chemical potential and study its Silver Blaze problem with nonperturbative lattice simulations employing stochastic quantization [14]. Stochastic quantization does not rely on the interpretation of $e^{-S}$ as a probability weight. Instead the proper distribution is obtained as the equilibrium distribution of a stochastic process, described by a Langevin equation. In the case that the action is complex, the Langevin dynamics is complexified [15, 16]. See Ref. 17] for a comprehensive review and Ref. [18] for an early application to the SU(3) spin model at finite chemical potential. In the past complex Langevin dynamics has been hindered by numerical instabilities and uncertainty about convergence, see e.g. Ref. [19]. Recently some of these problems were alleviated by the use of more refined Langevin algorithms 20, 21, 22, 23]. In particular, in Ref. 23] the method has been applied to several models at finite chemical potential. In $\mathrm{U}(1)$ and $\mathrm{SU}(3)$ one link models, where the sign problem is mild, excellent results have been obtained. For QCD with static quarks, first results on a $4^{4}$ lattice indicate a transition from a low-density confining to a high-density deconfining phase. Even though the sign problem appears to be severe, observables are under control. In this Letter we provide considerable evidence that stochastic quantization evades the sign problem and is capable of handling the Silver Blaze problem, in the context of the relativistic Bose gas at finite chemical potential.

Bose gas at finite chemical potential - We consider a self-interacting complex scalar field in $d=4$ euclidean dimensions, with the lattice action

$$
\begin{aligned}
S= & \sum_{x}\left[\left(2 d+m^{2}\right) \phi_{x}^{*} \phi_{x}+\lambda\left(\phi_{x}^{*} \phi_{x}\right)^{2}\right. \\
& \left.-\sum_{\nu=1}^{4}\left(\phi_{x}^{*} e^{-\mu \delta_{\nu, 4}} \phi_{x+\hat{\nu}}+\phi_{x+\hat{\nu}}^{*} e^{\mu \delta_{\nu, 4}} \phi_{x}\right)\right] .
\end{aligned}
$$


The lattice spacing $a=1$ and we take $m^{2}>0$. The lattice four-volume is $\Omega=N_{s}^{3} N_{\tau}$, where $N_{s}\left(N_{\tau}\right)$ are the number of sites in a spatial (temporal) direction. As usual, chemical potential $\mu$ is introduced as an imaginary constant vector potential in the temporal direction. Note that $S(\mu)=[S(-\mu)]^{*}$. At zero temperature and in the thermodynamic limit, bulk physical observables are strictly independent of the chemical potential as long as $\mu<\mu_{c}$, with $\mu_{c}$ the critical chemical potential. At $\mu=\mu_{c}$, one expects a second order phase transition to the Bose condensed phase, where the density $\langle n\rangle=(1 / \Omega) \partial \ln Z / \partial \mu$ is nonzero. Ignoring interactions, the critical chemical potential is given by $\left|\mu_{c}^{0}\right|=2 \operatorname{arcsinh}(m / 2)$ (corresponding to $\left|\mu_{c}^{0}\right|=m$ in the formal continuum limit). Interactions are expected to increase this value [29]. The exact $\mu$-independence when $\mu<\mu_{c}$, even though microscopically $\mu$ is manifestly present, is the Silver Blaze problem.

Complex Langevin dynamics - To apply stochastic quantization, we start with the Langevin equation

$$
\frac{\partial \phi_{x}(\theta)}{\partial \theta}=-\frac{\delta S[\phi]}{\delta \phi_{x}(\theta)}+\eta_{x}(\theta)
$$

where $\theta$ is the Langevin time and $\eta$ is Gaussian random noise. Since the action is complex, stochastic quantization relies on a complexification of the fields. The original field is first written in terms of two real fields $\phi_{a}(a=1,2)$ as $\phi=\frac{1}{\sqrt{2}}\left(\phi_{1}+i \phi_{2}\right)$. In terms of these the action reads

$$
\begin{aligned}
S= & \sum_{x}\left[\left(d+\frac{m^{2}}{2}\right) \phi_{a, x}^{2}+\frac{\lambda}{4}\left(\phi_{a, x}^{2}\right)^{2}-\sum_{i=1}^{3} \phi_{a, x} \phi_{a, x+\hat{i}}\right. \\
& \left.-\cosh \mu \phi_{a, x} \phi_{a, x+\hat{4}}+i \sinh \mu \varepsilon_{a b} \phi_{a, x} \phi_{b, x+\hat{4}}\right] . \quad \text { (3) }
\end{aligned}
$$

Here the antisymmetric tensor $\varepsilon_{a b}$, with $\varepsilon_{12}=-\varepsilon_{21}=$ 1, $\varepsilon_{11}=\varepsilon_{22}=0$, is introduced and summation over repeated indices is implied. The term proportional to $\sinh \mu$ causes the action to be complex.

These real fields are now complexified as

$$
\phi_{a} \rightarrow \phi_{a}^{\mathrm{R}}+i \phi_{a}^{\mathrm{I}} \quad(a=1,2),
$$

and the complex Langevin equations read

$$
\begin{aligned}
& \phi_{a, x}^{\mathrm{R}}(n+1)=\phi_{a, x}^{\mathrm{R}}(n)+\epsilon K_{a, x}^{\mathrm{R}}(n)+\sqrt{\epsilon} \eta_{a, x}(n), \\
& \phi_{a, x}^{\mathrm{I}}(n+1)=\phi_{a, x}^{\mathrm{I}}(n)+\epsilon K_{a, x}^{\mathrm{I}}(n) .
\end{aligned}
$$

Here Langevin time is discretized as $\theta=n \epsilon$, with $\epsilon$ the time step, the noise $\eta$ is real and Gaussian, with $\left\langle\eta_{a, x}(n)\right\rangle=0,\left\langle\eta_{a, x}(n) \eta_{b, x^{\prime}}\left(n^{\prime}\right)\right\rangle=2 \delta_{n n^{\prime}} \delta_{a b} \delta_{x x^{\prime}}$, and the drift terms are determined by

$$
\begin{aligned}
K_{a, x}^{\mathrm{R}} & =-\left.\operatorname{Re} \frac{\delta S}{\delta \phi_{a, x}}\right|_{\phi_{a} \rightarrow \phi_{a}^{\mathrm{R}}+i \phi_{a}^{\mathrm{I}}}, \\
K_{a, x}^{\mathrm{I}} & =-\left.\operatorname{Im} \frac{\delta S}{\delta \phi_{a, x}}\right|_{\phi_{a} \rightarrow \phi_{a}^{\mathrm{R}}+i \phi_{a}^{\mathrm{I}}} .
\end{aligned}
$$

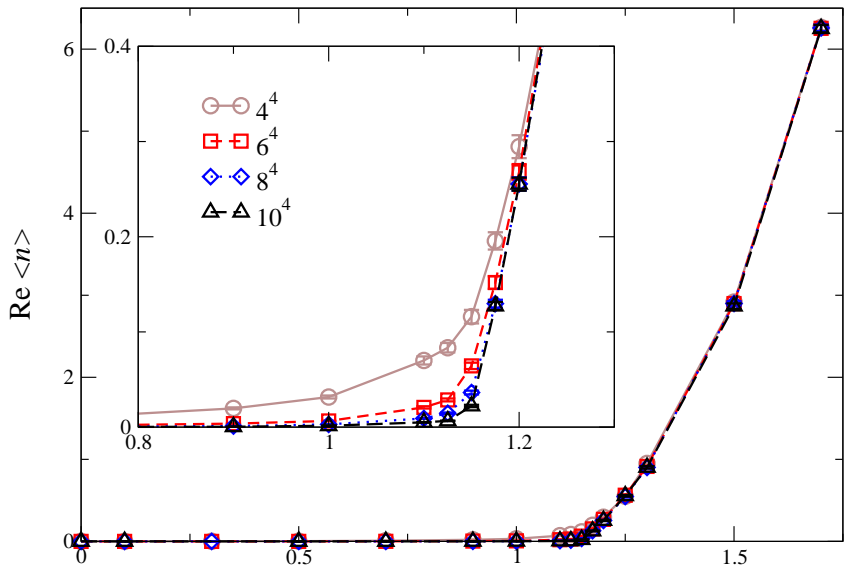

$\mu$

FIG. 1: Real part of the density, $\operatorname{Re}\langle n\rangle$, as a function of chemical potential for lattices of size $N^{4}$, with $N=4,6,8,10$. The parameters are $m=\lambda=1$ and stepsize $\epsilon=5 \times 10^{-5}$. The inset shows a blowup around the transition. In the thermodynamic limit, the density vanishes below the critical chemical potential.

Explicitly, they read

$$
\begin{aligned}
K_{a, x}^{\mathrm{R}}= & -\left[2 d+m^{2}+\lambda\left(\phi_{b, x}^{\mathrm{R} 2}-\phi_{b, x}^{\mathrm{I} 2}\right)\right] \phi_{a, x}^{\mathrm{R}} \\
& +2 \lambda \phi_{b, x}^{\mathrm{R}} \phi_{b, x}^{\mathrm{I}} \phi_{a, x}^{\mathrm{I}}+\sum_{i}\left(\phi_{a, x+\hat{i}}^{\mathrm{R}}+\phi_{a, x-\hat{i}}^{\mathrm{R}}\right) \\
& +\cosh \mu\left(\phi_{a, x+\hat{4}}^{\mathrm{R}}+\phi_{a, x-\hat{4}}^{\mathrm{R}}\right) \\
& +\sinh \mu \varepsilon_{a b}\left(\phi_{b, x+\hat{4}}^{\mathrm{I}}-\phi_{b, x-\hat{4}}^{\mathrm{I}}\right), \\
K_{a, x}^{\mathrm{I}}= & -\left[2 d+m^{2}+\lambda\left(\phi_{b, x}^{\mathrm{R} 2}-\phi_{b, x}^{\mathrm{I} 2}\right)\right] \phi_{a, x}^{\mathrm{I}} \\
& -2 \lambda \phi_{b, x}^{\mathrm{R}} \phi_{b, x}^{\mathrm{I}} \phi_{a, x}^{\mathrm{R}}+\sum_{i}\left(\phi_{a, x+\hat{i}}^{\mathrm{I}}+\phi_{a, x-\hat{i}}^{\mathrm{I}}\right) \\
& +\cosh \mu\left(\phi_{a, x+\hat{4}}^{\mathrm{I}}+\phi_{a, x-\hat{4}}^{\mathrm{I}}\right) \\
& -\sinh \mu \varepsilon_{a b}\left(\phi_{b, x+\hat{4}}^{\mathrm{R}}-\phi_{b, x-\hat{4}}^{\mathrm{R}}\right) .
\end{aligned}
$$

Observables are written in terms of the complexified fields using the replacement (44). For instance, the square of the field modulus is given by

$$
\phi^{*} \phi=\frac{1}{2} \phi_{a}^{2} \rightarrow \frac{1}{2}\left(\phi_{a}^{\mathrm{R}^{2}}-\phi_{a}^{\mathrm{I}}{ }^{2}\right)+i \phi_{a}^{\mathrm{R}} \phi_{a}^{\mathrm{I}},
$$

and the density is given by $n=\frac{1}{\Omega} \sum_{x} n_{x}$, with

$$
\begin{aligned}
n_{x}= & \left(\delta_{a b} \sinh \mu-i \varepsilon_{a b} \cosh \mu\right) \phi_{a, x} \phi_{b, x+\hat{4}} \\
\rightarrow & \left(\delta_{a b} \sinh \mu-i \varepsilon_{a b} \cosh \mu\right)\left(\phi_{a, x}^{\mathrm{R}} \phi_{b, x+\hat{4}}^{\mathrm{R}}-\phi_{a, x}^{\mathrm{I}} \phi_{b, x+\hat{4}}^{\mathrm{I}}\right. \\
& \left.+i\left[\phi_{a, x}^{\mathrm{R}} \phi_{b, x+\hat{4}}^{\mathrm{I}}+\phi_{a, x}^{\mathrm{I}} \phi_{b, x+\hat{4}}^{\mathrm{R}}\right]\right) .
\end{aligned}
$$

All observables now have a real and imaginary part.

Results - We have solved Eqs. (5, 6) numerically, using a Langevin stepsize $\epsilon=5 \times 10^{-5}$, a total number of 


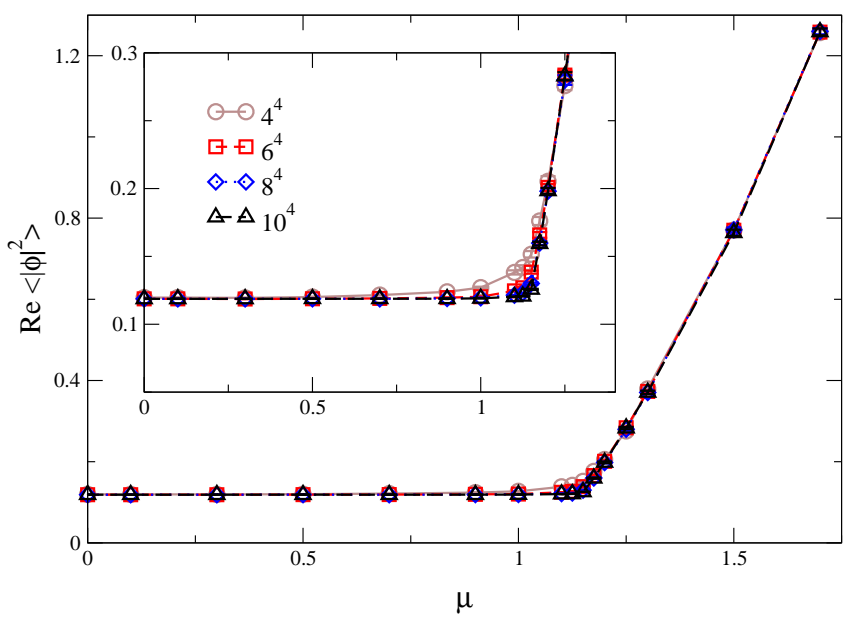

FIG. 2: Real part of the square of the field modulus, $\operatorname{Re}\left\langle|\phi|^{2}\right\rangle$, as a function of $\mu$. The inset shows a blowup at smaller $\mu$.

$5 \times 10^{6}$ Langevin timesteps, and $m=\lambda=1$. Lattices of size $N^{4}$, with $N=4,6,8,10$ were used. No instabilities or runaway solutions were encountered. The real part of the density is shown in Fig. 1 for chemical potentials $0 \leq \mu \leq 1.7$. Imaginary parts of observables were found to be consistent with zero, as they should. A transition between a zero-density phase and a condensed phase with nonzero density is clearly visible. The inset shows a blowup of the transition region. Nonanalyticity associated with a phase transition can only occur in the thermodynamic limit. We observe that with increasing four-volume the transition becomes sharper and the density goes to zero below $\mu_{c} \approx 1.15$, as is expected in a second order phase transition [30]. Finite size effects are comparable to what is found analytically in the noninteracting system, when $|\mu|<\left|\mu_{c}^{0}\right|$. In Fig. 2 we show the real part of the square of the field modulus (11). Again we observe $\mu$-independence below $\mu_{c}$ and a sharp rise above. We note that the value at $\mu=0$ is obtained using real Langevin dynamics and is therefore theoretically well established. We conclude that the Silver Blaze problem poses no obstacle for stochastic quantization and that there is no problem in taking the thermodynamic limit.

Some insight into why this method works can be obtained by ignoring the interactions $(\lambda=0)$ 25]. In this case the complex Langevin equations can be solved analytically and convergence to the exact results can be proven, provided that $|\mu|<\left|\mu_{c}^{0}\right| \quad(=0.9624$ for the parameters used here). For larger $\mu$, the free theory is unstable. As we have shown here, interactions shift the critical chemical potential and remove the instability.

Sign problem and phase quenching - To quantify the sign problem, we write $e^{-S}=e^{-S_{\mathrm{R}}-i S_{\mathrm{I}}}=\left|e^{-S}\right| e^{i \varphi}$. In reweighting, the phase factor $e^{i \varphi}$ is combined with the observable while simulations are performed in the phasequenched theory, obtained by ignoring the imaginary part of the action. Expectation values in the full the-

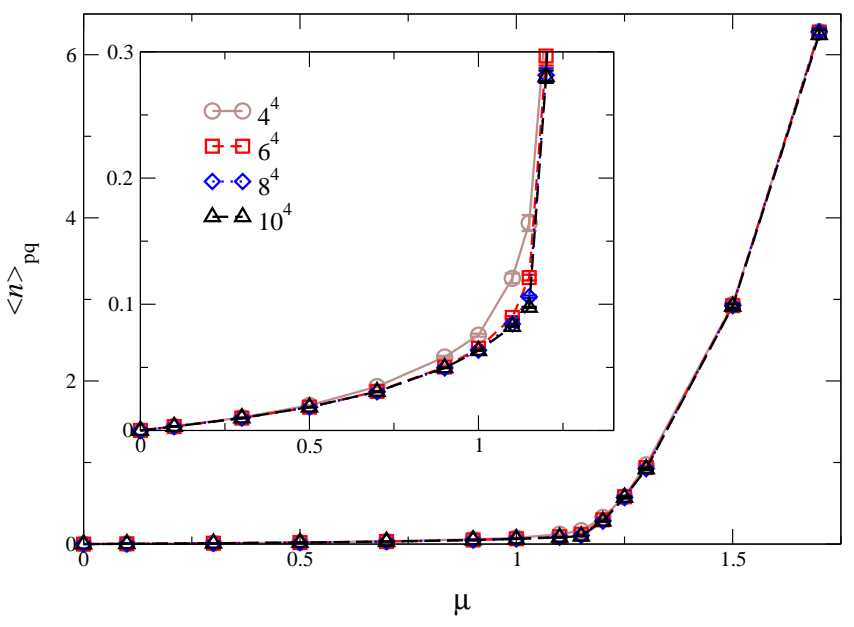

FIG. 3: Density in the phase-quenched theory, $\langle n\rangle_{\mathrm{pq}}$, as a function of $\mu$. The inset shows a blowup at smaller $\mu$.

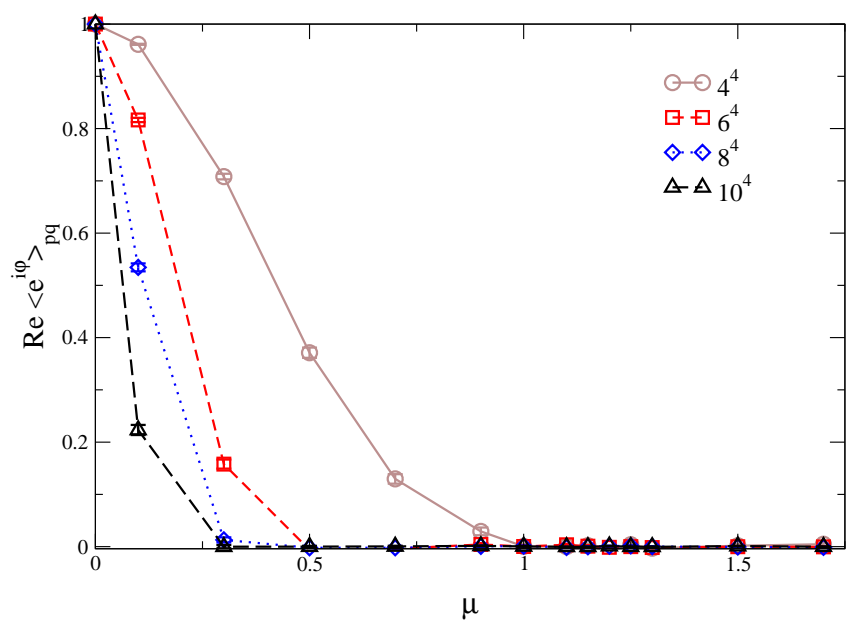

FIG. 4: Average phase factor in the phase-quenched theory, $\operatorname{Re}\left\langle e^{i \varphi}\right\rangle_{\mathrm{pq}}$, as a function of $\mu$, indicating the severeness of the sign problem in the thermodynamic limit.

ory are then reconstructed as $\langle O\rangle_{\mathrm{full}}=\left\langle O e^{i \varphi}\right\rangle_{\mathrm{pq}} /\left\langle e^{i \varphi}\right\rangle_{\mathrm{pq}}$, where the subscript pq indicates phase quenched. The average phase factor $\left\langle e^{i \varphi}\right\rangle_{\mathrm{pq}}$ is the ratio of two partition functions, $\left\langle e^{i \varphi}\right\rangle_{\mathrm{pq}}=Z_{\mathrm{full}} / Z_{\mathrm{pq}}=e^{-\Omega \Delta f}$, and vanishes exponentially in the thermodynamic limit $\Omega \rightarrow \infty$, when $\mu \neq 0$. Here $\Delta f$ is the difference between the free energy densities in the full and phase quenched theories.

We have studied the phase-quenched theory numerically. In this case, real Langevin dynamics can be employed, using only Eq. (5) with $\phi_{a}^{\mathrm{I}} \equiv 0$. In the expression for the density (12), only the term proportional to $\sinh \mu$ is preserved. Simulations were carried out using the same parameters as above. No difference in convergence properties were found. The density in the phase-quenched theory is shown in Fig. 3. We observe that the density increases linearly as soon as $\mu \neq 0$. This is not unexpected, since the density is proportional to $\sinh \mu$. However, it 
makes it even more remarkable that in the full theory all $\mu$ dependence precisely cancels. We note that this is similar to what is expected to occur in phase-quenched QCD when $m_{\pi} / 2<\mu<m_{N} / 3$ [26]. Beyond the critical chemical potential, the density increases rapidly in the phase-quenched theory as well.

We have also computed the average phase factor in the phase-quenched theory using real Langevin dynamics. The results are shown in Fig. 4, For large chemical potential the phase factor goes to zero on all lattices, making reweighting impossible. For small chemical potential, the phase factor goes to zero exponentially fast as the four-volume increases. This is precisely how the average phase factor is expected to behave [27]. However, we stress again that in the simulations of the full theory no negative impact of the sign problem was found.

Summary and outlook - We have applied stochastic quantization and complex Langevin dynamics to study the Silver Blaze problem in the relativistic Bose gas at finite chemical potential, both in the full and the phase quenched theory. We found precise agreement with theoretical expectations, and no obstacles related to the sign problem or in taking the thermodynamic limit. These results clearly stimulate the application of this approach to more complicated theories with a sign problem, in particular QCD at nonzero baryon density.

In this context two aspects have to be mentioned. First, due to the complexification, the Langevin dynamics no longer takes place in $\mathrm{SU}(3)$ but instead in $\mathrm{SL}(3$, $\mathbb{C})$. This has been discussed in detail in Ref. [23], where first numerical results for QCD with a nonzero density of static quarks can be found. Second, the inclusion of dynamical fermions, not discussed explicitly in Ref. [23], is relatively straightforward. After integrating out the fermion fields, the fermion determinant contributes to the force term for the gauge fields. This is not different from any conventional algorithm used in lattice QCD simulations, except that the force is now complex, making necessary the extension from $\mathrm{SU}(3)$ to $\mathrm{SL}(3, \mathbb{C})$. For details concerning the inclusion of fermions in Langevin dynamics, see e.g. Ref. [28]. Work in this direction is currently in progress.

Discussions with I.O. Stamatescu and S. Hands are greatly appreciated. This work is supported by STFC.

[1] Z. Fodor and S. D. Katz, Phys. Lett. B 534 (2002) 87 hep-lat/0104001; JHEP 0203 (2002) 014 hep-lat/0106002; JHEP $0404 \quad$ (2004) 050 hep-lat/0402006.

[2] Z. Fodor, S. D. Katz and K. K. Szabo, Phys. Lett. B 568 (2003) 73 hep-lat/0208078.

[3] C. R. Allton et al., Phys. Rev. D 66 (2002) 074507 hep-lat/0204010; $\quad$ ibid. 68 (2003)
014507 hep-lat/0305007; $\quad$ ibid. $\mathbf{7 1}$ (2005) 054508 hep-lat/0501030.

[4] R. V. Gavai and S. Gupta, Phys. Rev. D 68 (2003) 034506 hep-lat/0303013.

[5] P. de Forcrand and O. Philipsen, Nucl. Phys. B 642 (2002) 290 hep-lat/0205016; ibid. $\mathbf{6 7 3}$ (2003) 170 hep-lat/0307020; JHEP 0701 (2007) 077 hep-lat/0607017.

[6] M. D'Elia and M. P. Lombardo, Phys. Rev. D 67 (2003) 014505 hep-lat/0209146.

[7] S. Kratochvila and P. de Forcrand, PoS LAT2005 (2006) 167 hep-lat/0509143.

[8] S. Ejiri, arXiv:0804.3227 [hep-lat].

[9] Z. Fodor, S. D. Katz and C. Schmidt, JHEP 0703 (2007) 121 hep-lat/0701022.

[10] T. D. Cohen, Phys. Rev. Lett. 91 (2003) 222001 hep-ph/0307089.

[11] J. C. Osborn, Phys. Rev. Lett. 93 (2004) 222001 hep-th/0403131.

[12] J. C. Osborn, K. Splittorff and J. J. M. Verbaarschot, Phys. Rev. Lett. 94 (2005) 202001 hep-th/0501210.

[13] G. Akemann, J. C. Osborn, K. Splittorff and J. J. M. Verbaarschot, Nucl. Phys. B 712 (2005) 287 hep-th/0411030.

[14] G. Parisi and Y. s. Wu, Sci. Sin. 24 (1981) 483.

[15] G. Parisi, Phys. Lett. B 131 (1983) 393.

[16] J. R. Klauder and W. P. Petersen, SIAM J. Numer. Anal. 22 (1985) 1153; J. Stat. Phys. 39 (1985) 53.

[17] P. H. Damgaard and H. Huffel, Phys. Rept. 152 (1987) 227.

[18] F. Karsch and H. W. Wyld, Phys. Rev. Lett. 55 (1985) 2242 .

[19] J. Ambjorn, M. Flensburg and C. Peterson, Nucl. Phys. B 275 (1986) 375.

[20] J. Berges and I. O. Stamatescu, Phys. Rev. Lett. 95 (2005) 202003 hep-lat/0508030.

[21] J. Berges, S. Borsanyi, D. Sexty and I. O. Stamatescu, Phys. Rev. D 75 (2007) 045007 hep-lat/0609058.

[22] J. Berges and D. Sexty, Nucl. Phys. B 799 (2008) 306 [0708.0779 [hep-lat]].

[23] G. Aarts and I. O. Stamatescu, JHEP 0809 (2008) 018 [0807.1597 [hep-lat]].

[24] M. G. Endres, Phys. Rev. D 75 (2007) 065012 hep-lat/0610029.

[25] G. Aarts, arXiv:0902.4686 [hep-lat].

[26] D. T. Son and M. A. Stephanov, Phys. Rev. Lett. 86 (2001) 592 hep-ph/0005225.

[27] K. Splittorff and J. J. M. Verbaarschot, Phys. Rev. Lett. 98 (2007) 031601 hep-lat/0609076.

[28] G. G. Batrouni, G. R. Katz, A. S. Kronfeld, G. P. Lepage, B. Svetitsky and K. G. Wilson, Phys. Rev. D 32 (1985) 2736 .

[29] See e.g. Ref. [24]. In this paper a reformulation of this model in terms of dual lattice variables was given, which avoids the sign problem, and numerical results for two rather than three spatial dimensions were obtained.

[30] In this study we have not attempted to determine the precise value of $\mu_{c}$ or the order of the phase transition, which can be done using e.g. Binder cumulants. A mean field estimate of the critical chemical potential, $4 \sinh ^{2}\left(\mu_{c} / 2\right)=m^{2}+4 \lambda\left\langle|\phi|^{2}\right\rangle$, yields $\mu_{c}=1.15[25]$. 